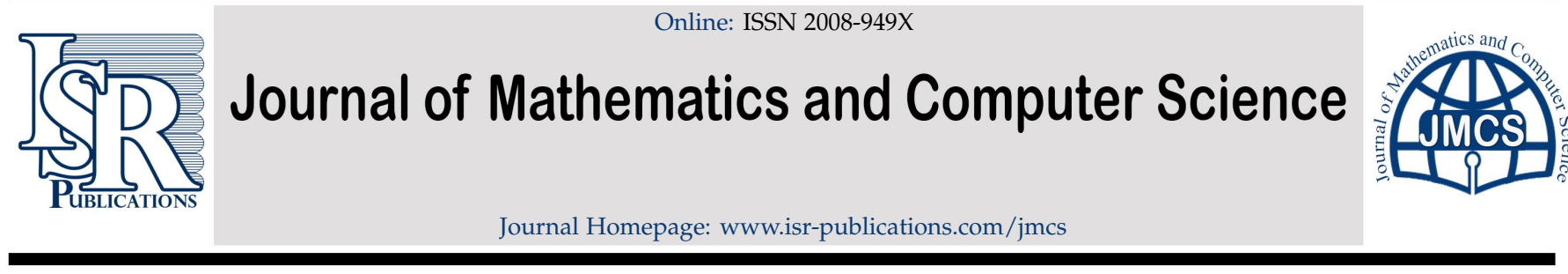

\title{
Spaces of neutrosophic $\lambda$-statistical convergence sequences and their properties
}

\author{
Vakeel A. Khan ${ }^{\mathrm{a}, *}$, Hira Fatima ${ }^{\mathrm{b}}$, Mohammad Daud Khan ${ }^{\mathrm{a}}$, Ayaz Ahamd ${ }^{\mathrm{C}}$ \\ ${ }^{a}$ Department of Mathematics, Aligarh Muslim University, Aligarh-202002, India. \\ ${ }^{b}$ Department of Institute of Applied Sciences, Mangalayatan University-202001, India. \\ ${ }^{c}$ Department of Mathematics, National Institute of Technology, Patna, India.
}

\begin{abstract}
In this paper, we use the notion of $\lambda$-statistical convergence in order to generalize these concepts. We establish some inclusion relations between them. We define the statistical convergence and $\lambda$-statistical convergence in neutrosophic normed space. We give the $\lambda$-statistically Cauchy sequence in neutrosophic normed space and present the $\lambda$-statistically completeness in connection with a neutrosophic normed space. Some interesting examples are also displayed here in support of our definitions and results.
\end{abstract}

Keywords: $t$-norm, $t$-conorm, neutrosophic normed space, statistical convergence, $\lambda$-statistical convergence, $\lambda$-statistical Cauchy.

2020 MSC: 46S40, 11B39, 03E72, 40G15.

(C)2021 All rights reserved.

\section{Introduction}

Fuzzy sets (FSs) put forward by Zadeh [16] has influenced deeply all the scientific fields since the publication of the paper. This concept is very important for real-life situations. Atanassov [1] initiated intuitionistic fuzzy sets (IFSs) for such cases. Neutrosophic set (NS) is a new version of the idea of the classical set which is defined by Smarandache [15]. Examples of generalizations of FS are interval-valued FS, IFS, interval-valued IFS, the sets paraconsistent, dialetheist, paradoxist, and tautological, Pythagorean fuzzy sets. One of the most important problems in fuzzy topology is to obtain an appropriate concept of fuzzy metric space. Park [13] introduced and discussed in a notion of intuitionistic fuzzy metric spaces which is based both on the idea of intuitionistic fuzzy set due to Atanassov [1] and the concept of a fuzzy metric space by George and Veeramani [10]. After defined of the intuitionistic fuzzy set (IFS), it was used in all areas where FS theory was studied. Park [13] defined IF metric space (IFMS), which is a generalization of FMSs. Park used George and Veeramani's [10] thought of applying t-norm and t-conorm to FMS meanwhile defining IFMS and studying its basic features. Esi and Hazarika [6], introduced $\lambda$ Ideal convergence in intuitionistic fuzzy 2-normed linear space. Bera and Mahapatra [2] defined the

\footnotetext{
${ }^{*}$ Corresponding author

Email address: vakhanmaths@gmail.com (Vakeel A. Khan)

doi: $10.22436 /$ jmcs.023.01.01
}

Received: 2020-06-20 Revised: 2020-08-07 Accepted: 2020-08-17 
neutrosophic soft linear spaces (NSLSs). In [2] neutrosophic norm, Cauchy sequence in NSNLS, convexity of NSNLS, metric in NSNLS were studied.

The notion of the statistical convergence was introduced by Fast [8]. Later on it was analyzed by Fridy [9] from the sequence point of view and linked it with the summability theory. Esi and Braha [5] examined on asymptotically statistical equivalent sequences of interval numbers. Esi [4] studied asymptotically $\lambda$ invariant statistical equivalent sequences of fuzzy numbers

\section{Definitions and preliminaries}

Definition 2.1. In terms of the set $\mathrm{N}$ of positive integers, let

$$
\left\{K \subseteq N \text { and } K_{n}=j: j \leqslant n \text { and } j \in K\right\} .
$$

Then the natural density of $\mathrm{K}$ is defined by

$$
\delta(K)=\lim _{n \rightarrow \infty} \frac{\left|K_{n}\right|}{n}
$$

if the limit exists, where $\left|K_{n}\right|$ denotes the cardinality of the set $K_{n}$.

Definition 2.2. A sequence $x=\left\{x_{k} ; k=1,2,3, \ldots\right\}$ is statistically convergent to $l$ if

$$
\left(\left\{k:\left|x_{k}-l\right| \geqslant \epsilon\right\}\right)=0
$$

for every $\epsilon>0$, we have

$$
\lim _{n} \frac{1}{n}\left\{j: j \leqslant n \text { and }\left|x_{j}-l\right| \geqslant \epsilon\right\}=0 .
$$

Example 2.3. Let us consider the sequence $x=\left\{x_{k} ; k=1,2,3 \ldots,\right\}$ whose terms are

$$
x_{k}= \begin{cases}k, & \text { if } k=n^{2}, n=1,2,3, \ldots, \\ \frac{1}{k}, & \text { otherwise } .\end{cases}
$$

The concept of $\lambda$-statistical convergence was introduced recently in [4] as follows.

Definition 2.4. Let $\lambda=\left(\lambda_{n}\right)$ be a non-decreasing sequence of positive numbers tending to $\infty$ such that $\lambda_{n+1} \leqslant \lambda_{n}+1$ and $\lambda_{1}=1$. Also let $I_{n}=\left[n-\lambda_{n}+1, n\right]$ and $K \subseteq \mathbb{N}$. Then the $\lambda$-density of $K$ is defined by

$$
\delta_{\lambda}(K)=\lim _{n \rightarrow \infty} \mid\left\{j: j \in I_{n} \text { and } j \in K\right\} \mid .
$$

It is clear that any finite subset of $\mathbb{N}$ has zero $\lambda$-density and $\delta_{\lambda}\left(K^{\mathfrak{c}}\right)=1-\delta_{\lambda}(\mathrm{K})$ does not hold for $0<\alpha<1$ in general[7], when $\lambda_{n}=n$, the $\lambda$-density reduces to the above-defined natural density.

Definition 2.5. A sequence $x=\left(x_{n}\right)$ is said to be $\lambda$-statistically convergent or $S_{\lambda}$-convergent to $l$ if for every $\epsilon>0$

$$
\lim _{n \rightarrow \infty} \frac{1}{\lambda_{n}}\left|\left\{k \in I_{n}:\left|x_{n}-l\right| \geqslant \epsilon\right\}\right|=0 .
$$

In this case we write $S_{\lambda}-\lim x=l$ or $x_{n} \rightarrow l\left(S_{\lambda}\right)$, and

$$
S_{\lambda}:\left\{x: \exists l \in \mathbb{R}, S_{\lambda}-\lim x=l\right\} .
$$

Remark 2.6. If $A_{n}=n$, then $S_{\lambda}$ is the same as $S$.

Remark 2.7. $\lambda$-statistical convergence is a special case of A-statistical convergence $[3,11]$ if the matrix $A=\left(a_{n k}\right)$ is taken as

$$
x_{k}= \begin{cases}\frac{1}{\lambda_{n}}, & \text { if } k \in I_{n}, \\ 0, & \text { if } k \notin I_{n} .\end{cases}
$$


Triangular norms (t-norms) (TN) were initiated by Menger [12] . Triangular conorms (t-conorms) (TC) are known as dual operations of TNs. TNs and TCs are very significant for fuzzy operations (intersections and unions).

Definition 2.8 ([14]). A binary operation $*:[0,1] \times[0,1] \longrightarrow[0,1]$ is said to be a continuous t-norm if it satisfies the following conditions:

(a) $*$ is associative and commutative;

(b) $*$ is continuous;

(c) $a * 1=a$ for all $a \in[0,1]$;

(d) $a * b \leqslant c * d$ whenever $a \leqslant c$ and $b \leqslant d$ for each $a, b, c, d \in[0,1]$.

Definition 2.9 ([14]). A binary operation $\diamond:[0,1] \times[0,1] \longrightarrow[0,1]$ is said to be a continuous t-conorm if it satisfies the following conditions:

(a) $\diamond$ is associative and commutative;

(b) $\diamond$ is continuous;

(c) $a \diamond 0=a$ for all $a \in[0,1]$;

(d) $a \diamond b \leqslant c \diamond d$ whenever $a \leqslant c$ and $b \leqslant d$ for each $a, b, c, d \in[0,1]$.

Form above definitions, we note that if we choose $0<e_{1}, e_{2}<1$ for $e_{1}>e_{2}$, then there exist $0<e_{3}, e_{4}<$ 0,1 such that $e_{1} * e_{3} \geqslant e_{2}, e_{1} \geqslant e_{4} \diamond e_{2}$. Further, if we choose $e_{5} \in(0,1)$, then there exist $e_{6}, e_{7} \in(0,1)$ such that $e_{6} * e_{6} \geqslant e_{5}$ and $e_{7} \diamond e_{7} \leqslant e_{5}$.

In this paper, neutrosophic normed space (NNS) is defined and the definition statistical convergence with respect to NNS is given. The fundamental properties of NNS and statistical convergence with respect to NNS are investigated.

Definition 2.10 ([2]). Take $F$ as a vector space, $N=\{\langle u, \mathcal{G}(u), \mathcal{B}(u), y(u)\rangle: u \in F\}$ be a normed space(NS) such that $\mathrm{N}: \mathrm{F} \times \mathbb{R}^{+} \rightarrow[0,1]$ show continuous $\mathrm{TN}$ and continuous $\mathrm{TC}$, respectively. If the following conditions are hold, then the four-tuple $V=(F, N, *, \diamond)$ is called NNS, for all $u, v \in F$ and $\beta, \mu>0$ and for each $\sigma \neq 0$,
(a) $0 \leqslant \mathcal{G}(u, \beta) \leqslant 1,0 \leqslant \mathcal{B}(u, \beta) \leqslant 1,0 \leqslant y(u, \beta) \leqslant 1$;
(b) $\mathcal{G}(u, \beta)+\mathcal{B}(u, \beta)+y(u, \beta) \leqslant 3, \quad \beta \in \mathbb{R}^{+}$;
(c) $\mathcal{G}(u, \beta)=1$ (for $\beta>0$ ) if and only if $u=0$;
(d) $\mathcal{G}(\sigma u, \beta)=\mathcal{G}\left(u, \frac{\beta}{|\sigma|}\right)$ for each $\sigma \neq 0$;
(e) $\mathcal{G}(u, \beta) * \mathcal{G}(v, \mu) \leqslant \mathcal{G}(u+v, \beta+\mu)$;
(f) $\mathcal{G}(u,$.$) is continuous non-decreasing function;$
(g) $\lim _{\beta \rightarrow \infty} \mathcal{G}(u, \beta)=1$;
(h) $\mathcal{B}(u, \beta)=0$ (for $\beta>0$ ) if and only if $u=0$;
(i) $\mathcal{B}(\sigma u, \beta)=\mathcal{B}\left(u, \frac{\beta}{|\sigma|}\right)$ for each $\sigma \neq 0$;
(j) $\mathcal{B}(u, \beta) * \mathcal{B}(v, \mu) \geqslant \mathcal{B}(u+v, \beta+\mu)$;
(k) $\mathcal{B}(u,$.$) is continuous non-increasing function;$
(l) $\lim _{\beta \rightarrow \infty} \mathcal{B}(u, \beta)=1$;

(m) $y(u, \beta)=0$ (for $\beta>0$ ) if and only if $u=0$;

(n) $y(\sigma u, \beta)=y\left(u, \frac{\beta}{|\sigma|}\right)$ for each $\sigma \neq 0$;

(o) $y(u, \beta) * y(v, \mu) \geqslant y(u+v, \beta+\mu)$;

(p) $y(u,$.$) is continuous non-increasing function;$

(q) $\lim _{\beta \rightarrow \infty} y(u, \beta)=1$;

(r) if $\beta \leqslant 0$, then $\mathcal{G}(u, \beta)=0, \mathcal{B}(u, \beta)=1$ and $y(u, \beta)=1$. 
Then $(N, \mathcal{G}, \mathcal{B}, y)$ is called neutrosophic norm $(\mathrm{NN})$.

Example 2.11. Let $(\mathrm{F},\|\|$.$) be a normed space. Give the operations *$ and $\diamond$ being $\mathrm{TN}$ and TC, respectively, and $u * v=u v, u \diamond v=u+v-u v$. For $\beta>\|u\|$,

$$
\mathcal{G}(u, \beta)=\frac{\beta}{\beta+\|u\|}, \mathcal{B}(u, \beta)=\frac{\|u\|}{\beta+\|u\|}, y(u, \beta)=\frac{\|u\|}{\beta}
$$

for all $u, v \in F$ and $\beta>0$. If we take $\beta \leqslant\|u\|$, then $\mathcal{G}(u, \beta)=0, \mathcal{B}(u, \beta)=1$ and $y(u, \beta)=1$. Then $(\mathrm{F}, \mathrm{N}, *, \diamond)$ is NNS such that $\mathrm{N}: \mathrm{F} \times \mathbb{R}^{+} \rightarrow[0,1]$.

Definition 2.12. Let $V$ be a neutrosophic normed space (NNS), and let the sequence $\left(x_{k}\right)$ in $V, 0<\epsilon<1$ and $\beta>0$. Then, the sequence $\left(x_{k}\right)$ is convergence to $l$ if and only if there exists $n \in \mathbb{N}$, such that $\mathcal{G}\left(x_{k}-l, \beta\right)>1-\epsilon, \mathcal{B}\left(x_{k}-l, \beta\right)<\epsilon, y\left(x_{k}-l, \beta\right)<\epsilon$. That is $\lim _{k \rightarrow \infty} \mathcal{G}\left(x_{k}-l, \beta\right)=1, \lim _{k \rightarrow \infty} \mathcal{B}\left(x_{k}-l, \beta\right)=0$, $\lim _{k \rightarrow \infty} y\left(x_{k}-l, \beta\right)=0$. In that case, the sequence $\left(x_{k}\right)$ is called a convergent sequence in $V$. The convergent in NNS is denoted by $\mathrm{N}-\lim x_{k}=l$.

Definition 2.13. Take a NNS V. A sequence $\left(x_{k}\right)$ is called statistical convergence with respect to NN (SC-NN), if there exist $l \in F$ such that the set

$$
K_{\epsilon}:=\left\{k \leqslant n: \mathcal{G}\left(x_{k}-l, \beta\right) \leqslant 1-\epsilon \text { or } \mathcal{B}\left(x_{k}-l, \beta\right) \geqslant \epsilon, y\left(x_{k}-l, \beta\right) \geqslant \epsilon\right\}
$$

has ND zero, for every $\epsilon>0$ and $\beta>0$ or equivalently,

$$
\lim _{n} \frac{1}{n} \mid\left\{k \leqslant n: \mathcal{G}\left(x_{k}-l, \beta\right) \leqslant 1-\epsilon \text { or } \mathcal{B}\left(x_{k}-l, \beta\right) \geqslant \epsilon, y\left(x_{k}-l, \beta\right) \geqslant \epsilon\right\} \mid=0 .
$$

Therefore, we write $S_{N}-\lim x_{k}=l$ or $x_{k} \rightarrow l\left(S_{N}\right)$. The set of SC-NN will be denoted by SN. If $l=0$, then we will write $S_{0} N$.

\section{3. $\lambda$-statistical convergence on NNS}

In this section, we introduce the $\lambda$-statistical convergence on NNS which are needed in our subsequent discussions:

Definition 3.1. Let $\lambda=\left(\lambda_{n}\right)$ be a non-decreasing sequence of positive numbers tending to infinity such that $\lambda_{n+1} \leqslant \lambda_{n}+1$ and $\lambda_{1}=1$. Also let $I_{n}=\left[n-\lambda_{n}+1, n\right]$. Take a NNS $V$. A sequence $\left(x_{k}\right)$ is called $\lambda$-statistical convergence with respect to $\mathrm{NN}$ (SC-NN), if there exist $l \in \mathrm{F}$ such that the set

$$
{ }_{\lambda} K_{\epsilon}:=\left\{k \in I_{n}: \mathcal{G}\left(x_{k}-l, \beta\right) \leqslant 1-\epsilon \text { or } \mathcal{B}\left(x_{k}-l, \beta\right) \geqslant \epsilon, y\left(x_{k}-l, \beta\right) \geqslant \epsilon\right\}
$$

has $\lambda$-density zero, for every $\epsilon>0$ and $\beta>0$ or equivalently,

$$
\lim _{n} \frac{1}{\lambda_{n}} \mid\left\{k \in I_{n}: \mathcal{G}\left(x_{k}-l, \beta\right) \leqslant 1-\epsilon \text { or } \mathcal{B}\left(x_{k}-l, \beta\right) \geqslant \epsilon, y\left(x_{k}-l, \beta\right) \geqslant \epsilon\right\} \mid=0 .
$$

Therefore, we write ${ }_{\lambda} S_{N}-\lim x_{k}=l$ or $x_{k} \rightarrow l\left({ }_{\lambda} S_{N}\right)$. The set of $\lambda-S C-N N$ will be denoted by ${ }_{\lambda} S_{N}$. If $l=0$, then we will write ${ }_{\lambda} S_{0} N$.

Example 3.2. Let $(F,\|\|$.$) be a NS. For all u, v \in[0,1]$, define the $\operatorname{TN} u * v=u v$ and $\operatorname{TC} u \diamond v=\min \{u+v, 1\}$. We take

$$
\mathcal{G}(u, \beta)=\frac{\beta}{\beta+\|u\|}, \mathcal{B}(u, \beta)=\frac{\|u\|}{\beta+\|u\|}, y(u, \beta)=\frac{\|u\|}{\beta}
$$

for all $u \in F, \beta>0$. Then, $V$ is a NNS. Define,

$$
x_{k}= \begin{cases}1, & \text { if } k=m^{2}(m \in \mathbb{N}), \\ 0, & \text { otherwise }\end{cases}
$$


Let $\lambda=\left(\lambda_{n}\right)$ be a non-decreasing sequence of positive numbers tending to $\infty$ such that $\lambda_{n+1} \leqslant$ $\lambda_{n}+1$ and $\lambda_{1}=1$. Also let $I_{n}=\left[n-\lambda_{n}+1, n\right]$. Consider

$$
{ }_{\lambda} K_{n}(\epsilon, \beta)=\left\{k \in I_{n}: \mathcal{G}(u, \beta) \leqslant 1-\epsilon \text { or } \mathcal{B}(u, \beta) \geqslant \epsilon, y(u, \beta) \geqslant \epsilon\right\}
$$

for every $\epsilon \in(0,1)$ and for any $\beta>0$. Then, we have

$$
\begin{aligned}
{ }_{\lambda} \mathrm{K}_{\mathrm{n}}(\epsilon, \beta) & =\left\{k \in I_{\mathrm{n}}: \frac{\beta}{\beta+\|\mathrm{u}\|} \leqslant 1-\epsilon \text { or } \frac{\|\mathrm{u}\|}{\beta+\|\mathrm{u}\|} \geqslant \epsilon, \frac{\|\mathrm{u}\|}{\beta} \geqslant \epsilon\right\} \\
& =\left\{k \in I_{\mathrm{n}}:\|\mathrm{u}\| \geqslant \frac{\beta \epsilon}{1-\epsilon} \text { or }\|\mathrm{u}\| \geqslant \beta \epsilon\right\} \\
& =\left\{k \in I_{\mathrm{n}}: x_{k}=1\right\}=\left\{k \leqslant n: k=m^{2} \text { and } m \in \mathbb{N}\right\} \leqslant \frac{\sqrt{\mathrm{n}}}{\mathrm{n}} .
\end{aligned}
$$

That is, when $n$ becomes sufficiently large, quantity $\mathcal{G}\left(x_{k}, \beta\right)$ becomes less that $1-\epsilon$ and similarly the quantities $\mathcal{B}\left(x_{k}, \beta\right)$ and $y\left(x_{k}, \beta\right)$ become larger than $\epsilon$. So

$$
\frac{1}{\lambda_{n}}\left|{ }_{\lambda} K_{n}(\epsilon, \beta)\right|=0 \text { for } \epsilon>0 \text { and } \beta>0 \text {. }
$$

Lemma 3.3 may be easily obtained by using the definitions and properties of $\lambda$-density.

Lemma 3.3. Choose a NNS V. The following statements are equivalent, for every $\epsilon>0$ and $\beta>0$ :

1. ${ }_{\lambda} S_{\mathrm{N}}-\lim x_{\mathrm{k}}=\mathrm{l}$;

2. $\lim _{n} \frac{1}{\lambda_{n}}\left|\left\{k \in I_{n}: \mathcal{G}\left(x_{k}-l, \beta\right) \leqslant 1-\epsilon\right\}\right|=\lim _{n} \frac{1}{\lambda_{n}}\left|\left\{k \in I_{n}: \mathcal{B}\left(x_{k}-l, \beta\right) \geqslant \epsilon\right\}\right|=\lim _{n} \frac{1}{\lambda_{n}} \mid\left\{k \in I_{n}:\right.$ $\left.y\left(x_{k}-l, \beta\right) \leqslant 1-\epsilon\right\} \mid=0$;

3. $\lim _{n} \frac{1}{\lambda_{n}} \mid\left\{k \in I_{n}: \mathcal{G}\left(x_{k}-l, \beta\right)>1-\epsilon\right.$ and $\left.\mathcal{B}\left(x_{k}-l, \beta\right)<\epsilon, y\left(x_{k}-l, \beta\right)>1-\epsilon\right\} \mid=1$;

4. ${ }_{\lambda} S-\lim \mathcal{G}\left(x_{k}-l, \beta\right)=1$, and ${ }_{\lambda} S-\lim \mathcal{B}\left(x_{k}-l, \beta\right)=0,{ }_{\lambda} S-\lim y\left(x_{k}-l, \beta\right)=0$.

Theorem 3.4. Let $\mathrm{V}$ be a NNS. If $\left(\mathrm{x}_{\mathrm{k}}\right)$ is $\lambda$-statistical convergent in neutrosophic normed space, then ${ }_{\lambda} S-\lim x_{k}=$ $l$ is unique.

Proof. Let $\lambda=\left(\lambda_{n}\right)$ be a non-decreasing sequence of positive numbers tending to infinity such that $\lambda_{n+1} \leqslant$ $\lambda_{n}+1$ and $\lambda_{1}=1$. Also let $I_{n}=\left[n-\lambda_{n}+1, n\right]$. Suppose that $S_{\lambda}-\lim x_{k}=l_{1}$ and $S_{\lambda}-\lim x_{k}=$ $l_{2}$, for $l_{1} \neq l_{2}$. Choose $\epsilon>0$. Then, for a given $\mu>0$ such that $(1-\epsilon) *(1-\epsilon)>1-\mu$ and $\epsilon \diamond \epsilon<\mu$. Then for any $\beta>0$, define the following sets as:

$$
\begin{array}{ll}
K_{\mathcal{G}_{1}}(\epsilon, \beta)=\left|\left\{k \in I_{n}: \mathcal{G}\left(x_{k}-l_{1}, \frac{\beta}{2}\right) \leqslant 1-\epsilon\right\}\right|=0, & K_{\mathcal{G}_{2}}(\epsilon, \beta)=\left|\left\{k \in I_{n}: \mathcal{G}\left(x_{k}-l_{2}, \frac{\beta}{2}\right) \leqslant 1-\epsilon\right\}\right|=0, \\
K_{\mathcal{B}_{1}}(\epsilon, \beta)=\left|\left\{k \in I_{n}: \mathcal{B}\left(x_{k}-l_{1}, \frac{\beta}{2}\right) \geqslant \epsilon\right\}\right|=0, & K_{\mathcal{B}_{2}}(\epsilon, \beta)=\left|\left\{k \in I_{n}: \mathcal{B}\left(x_{k}-l_{2}, \frac{\beta}{2}\right) \geqslant \epsilon\right\}\right|=0, \\
K_{y_{1}}(\epsilon, \beta)=\left|\left\{k \in I_{n}: y\left(x_{k}-l_{1}, \frac{\beta}{2}\right) \geqslant \epsilon\right\}\right|=0, & K_{y_{2}}(\epsilon, \beta)=\left|\left\{k \in I_{n}: y\left(x_{k}-l_{2}, \frac{\beta}{2}\right) \geqslant \epsilon\right\}\right|=0 .
\end{array}
$$

We know that $S_{\lambda}-\lim x_{k}=l_{1}$. Then, using the Lemma 3.3, for all $\beta>0$

$$
\delta_{\lambda}\left(K_{\mathcal{G}_{1}}(\epsilon, \beta)\right)=\delta_{\lambda}\left(K_{\mathcal{B}_{1}}(\epsilon, \beta)\right)=\delta_{\lambda}\left(K_{y_{1}}(\epsilon, \beta)\right)=0 .
$$

Further since $S_{\lambda}-\lim x_{k}=l_{2}$, then, using the Lemma 3.3, for all $\beta>0$

$$
\delta_{\lambda}\left(K_{\mathcal{G}_{2}}(\epsilon, \beta)\right)=\delta_{\lambda}\left(K_{\mathcal{B}_{2}}(\epsilon, \beta)\right)=\delta_{\lambda}\left(K_{y_{2}}(\epsilon, \beta)\right)=0 .
$$

Let

$$
\mathrm{K}_{\lambda}(\epsilon, \beta):=\left\{\mathrm{K}_{\mathcal{G}_{1}}(\epsilon, \beta) \cup \mathrm{K}_{\mathcal{G}_{2}}(\epsilon, \beta)\right\} \cap\left\{\mathrm{K}_{\mathcal{B}_{1}}(\epsilon, \beta) \cup \mathrm{K}_{\mathcal{B}_{2}}(\epsilon, \beta)\right\} \cap\left\{\mathrm{K}_{y_{1}}(\epsilon, \beta) \cup \mathrm{K}_{y_{2}}(\epsilon, \beta)\right\} .
$$

Then, observe that $\delta_{\lambda}\left(K_{\lambda}(\epsilon, \beta)\right)=0$, which implies $\delta_{\lambda}\left(\mathbb{N} / K_{\lambda}(\epsilon, \beta)\right)=1$. We have three possible situations, when take $k \in \mathbb{N} / K_{\lambda}(\epsilon, \beta)$ : 
(i) $\mathrm{k} \in \mathbb{N} /\left\{\mathrm{K}_{\mathcal{G}_{1}}(\epsilon, \beta) \cup \mathrm{K}_{\mathcal{G}_{2}}(\epsilon, \beta)\right\}$;

(ii) $\mathrm{k} \in \mathbb{N} /\left\{\mathrm{K}_{\mathcal{B}_{1}}(\epsilon, \beta) \cup \mathrm{K}_{\mathcal{B}_{2}}(\epsilon, \beta)\right\}$;

(iii) $\mathrm{k} \in \mathbb{N} /\left\{\mathrm{K}_{y_{1}}(\epsilon, \beta) \cup \mathrm{K}_{y_{2}}(\epsilon, \beta)\right\}$.

Firstly, consider (i). Then, we have

$$
\mathcal{G}\left(l_{1}-l_{2}, \beta\right) \geqslant \mathcal{G}\left(x_{k}-l_{1}, \frac{\beta}{2}\right) * \mathcal{G}\left(x_{k}-l_{2}, \frac{\beta}{2}\right)>(1-\epsilon) *(1-\epsilon)>1-\mu .
$$

Using the equation (3.1), for all $\beta>0$, we obtain $\mathcal{G}\left(l_{1}-l_{2}, \beta\right)=1$, where $\mu>0$ is arbitrary. That is, $l_{1}=l_{2}$ is obtained.

For the situation (ii), if we take $k \in \mathbb{N} /\left\{K_{\mathcal{B}_{1}}(\epsilon, \beta) \cup \mathrm{K}_{\mathcal{B}_{2}}(\epsilon, \beta)\right\}$, then, we can write

$$
\mathcal{B}\left(l_{1}-l_{2}, \beta\right) \leqslant \mathcal{B}\left(x_{k}-l_{1}, \frac{\beta}{2}\right) \diamond \mathcal{B}\left(x_{k}-l_{2}, \frac{\beta}{2}\right)<\epsilon \diamond \epsilon<\mu
$$

for all $\beta>0$, we obtain $\mathcal{B}\left(l_{1}-l_{2}, \beta\right)=0$, where $\mu>0$ is arbitrary. That is, $l_{1}=l_{2}$ is obtained. Again for the situation (iii), if we take $k \in \mathbb{N} /\left\{K_{y_{1}}(\epsilon, \beta) \cup K_{y_{2}}(\epsilon, \beta)\right\}$, then, we can write

$$
y\left(l_{1}-l_{2}, \beta\right) \leqslant y\left(x_{k}-l_{1}, \frac{\beta}{2}\right) \diamond y\left(x_{k}-l_{2}, \frac{\beta}{2}\right)<\epsilon \diamond \epsilon<\mu
$$

for all $\beta>0$, we obtain $y\left(l_{1}-l_{2}, \beta\right)=0$, where $\mu>0$ is arbitrary. That is, $l_{1}=l_{2}$ is obtained. This step completes the proof.

Theorem 3.5. If $\lim x_{k}=l$ for neutrosophic normed space (NNS) $\mathrm{V}$, then $S_{\lambda}-\lim x_{k}=l$.

Proof. If $\lim x_{k}=l$ for NNS $V$ then, for every $\epsilon>0$ and $\beta>0$, there exist a number $n \in \mathbb{N}$ such that $\mathcal{G}\left(x_{k}-l, \beta\right)>1-\epsilon, \mathcal{B}\left(x_{k}-l, \beta\right)<1-\epsilon$ and $y\left(x_{k}-l, \beta\right)<1-\epsilon$, for all $k \geqslant n$. Therefore, the set

$$
\left\{k \leqslant n: \mathcal{G}\left(x_{k}-l, \beta\right) \leqslant 1-\epsilon, \mathcal{B}\left(x_{k}-l, \beta\right) \geqslant 1-\epsilon \text { or } y\left(x_{k}-l, \beta\right) \geqslant 1-\epsilon\right\}
$$

so that we have

$$
\left\{k \in I_{n}: \mathcal{G}\left(x_{k}-l, \beta\right) \leqslant 1-\epsilon, \mathcal{B}\left(x_{k}-l, \beta\right) \geqslant 1-\epsilon \text { or } y\left(x_{k}-l, \beta\right) \geqslant 1-\epsilon\right\}
$$

and has at most finitely many terms, where $I_{n}=\left[n-\lambda_{n}+1, n\right]$ and $\left(\lambda_{n}\right)$ be a non-decreasing sequence of positive numbers tending to infinity such that $\lambda_{n+1} \leqslant \lambda_{n}+1$ and $\lambda_{1}=1$. Hence, since every finite subset of $\mathbb{N}$ has $\lambda$-density zero,

$$
\begin{aligned}
& \lim _{n} \frac{1}{\lambda_{n}} \mid\left\{n-\lambda_{n}+1 \leqslant k \leqslant n: \mathcal{G}\left(x_{k}-l, \beta\right) \leqslant 1-\epsilon,\right. \\
& \left.\mathcal{B}\left(x_{k}-l, \beta\right) \geqslant 1-\epsilon \text { or } y\left(x_{k}-l, \beta\right) \geqslant 1-\epsilon\right\} \mid=0 .
\end{aligned}
$$

This clearly shows that $S_{\lambda}-\lim x_{k}=l$.

Theorem 3.6. Let $\mathrm{V}$ be an NNS. ${ }_{\lambda} S_{N}-\lim x_{k}=$ liff there exists an increasing index sequence $\mathrm{J}=\left\{\mathrm{j}_{1}, j_{2}, \ldots\right\} \subseteq \mathbb{N}$ while $\delta_{\lambda}(J)=1$, then $\mathrm{N}-\lim _{\mathrm{n}} \mathrm{x}_{\mathbf{j}_{\mathrm{n}}}=\mathrm{l}$.

Proof. Suppose that ${ }_{\lambda} S_{N}-\lim x_{k}=l$. For any $\beta>0$ and $\mu=1,2,3, \ldots$

$$
P_{\lambda}(\mu, \beta)=\left\{k \leqslant n: \mathcal{G}\left(x_{k}-l, \beta\right)>1-\frac{1}{\mu} \text { and } \mathcal{B}\left(x_{k}-l, \beta\right)<\frac{1}{\mu}, y\left(x_{k}-l, \beta\right)<\frac{1}{\mu}\right\}
$$

and

$$
R_{\lambda}(\mu, \beta)=\left\{k \leqslant n: \mathcal{G}\left(x_{k}-l, \beta\right) \leqslant 1-\frac{1}{\mu} \text { and } \mathcal{B}\left(x_{k}-l, \beta\right) \geqslant \frac{1}{\mu}, y\left(x_{k}-l, \beta\right) \geqslant \frac{1}{\mu}\right\} .
$$


Then, $\delta_{\lambda}\left(R_{\lambda}(\mu, \beta)\right)=0$, since ${ }_{\lambda} S_{N}-\lim x_{k}=l$. Further, for $\beta>0$ and $\mu=1,2,3 \ldots$

$$
P_{\lambda}(\mu, \beta) \supset P_{\lambda}(\mu+1, \beta)
$$

and so,

$$
\delta_{\lambda}\left(P_{\lambda}(\mu, \beta)\right)=1 .
$$

Now, we will show that for $k \in P_{\lambda}(\mu, \beta), N-\lim x_{k}=l$. Assume that $N-\lim x_{k} \neq l$ for some $k \in P_{\lambda}(\mu, \beta)$. Then, there is $\rho>0$ and a positive integer $n$ such that $\mathcal{G}\left(x_{k}-l, \beta\right) \leqslant 1-\rho$ or $\mathcal{B}\left(x_{k}-l, \beta\right) \geqslant \rho, y\left(x_{k}-l, \beta\right) \geqslant$ $\rho$ for all $k \geqslant n$. Let $\mathcal{G}\left(x_{k}-l, \beta\right)>1-\rho$ or $\mathcal{B}\left(x_{k}-l, \beta\right)<\rho, y\left(x_{k}-l, \beta\right)<\rho$ for all $k \in I_{n}$, where $I_{n}=\left[n-\lambda_{n}+1, n\right]$ and $\lambda=\left(\lambda_{n}\right)$ be a non-decreasing sequence of positive numbers tending to infinity such that $\lambda_{n+1} \leqslant \lambda_{n}+1$ and $\lambda_{1}=1$. Hence

$$
\lim _{n} \frac{1}{\lambda_{n}} \mid\left\{k \in I_{n}: \mathcal{G}\left(x_{k}-l, \beta\right)>1-\rho \text { or } \mathcal{B}\left(x_{k}-l, \beta\right)<\rho, y\left(x_{k}-l, \beta\right)<\rho\right\} \mid=0 .
$$

Since $\rho>\frac{1}{\mu}$, we obtain $\delta_{\lambda}\left(P_{\lambda}(\mu, \beta)\right)=0$, which contradicts (3.2). That's why, $N-\lim x_{k}=l$. Assume that there exists a increasing index sequence $J=\left\{j_{1}, j_{2}, \ldots\right\} \subseteq \mathbb{N}$ while $\delta_{\lambda}(J)=1$, then $N-\lim _{n} x_{j_{n}}=l$, i.e., there exists a $n \in \mathbb{N}$ such that $\mathcal{G}\left(x_{k}-l, \beta\right)>1-\mu, \mathcal{B}\left(x_{k}-l, \beta\right)<\mu, y\left(x_{k}-l, \beta\right)<\mu$ for every $\mu>0$ and $\beta>0$. In that case

$$
R_{\lambda}(\mu, \beta)=\left\{k \in I_{n}: \mathcal{G}\left(x_{k}-l, \beta\right) \leqslant 1-\mu \text { and } \mathcal{B}\left(x_{k}-l, \beta\right) \geqslant \mu, y\left(x_{k}-l, \beta\right) \geqslant \mu\right\} \subseteq n-\left\{j_{n+1}, j_{n+2}, \ldots\right\} .
$$

Therefore $\delta_{\lambda}\left(R_{\lambda}(\mu, \beta)\right) \leqslant 1-1=0$, hence ${ }_{\lambda} S_{N}-\lim x_{k}=l$.

\section{4. $\lambda$-statistically complete NNS}

Definition 4.1. The sequence $\left(x_{k}\right)$ is called $\lambda$-statistically Cauchy with respect to neutrosophic norm (NN) in neutrosophic normed space(NNS) $V$, if there exists $n=n(\epsilon)$, for every $\epsilon>0$ and $\beta>0$ such that

$$
{ }_{\lambda} K C_{\epsilon}=\left\{k \in I_{n}: \mathcal{G}\left(x_{k}-x_{n}, \beta\right) \leqslant 1-\epsilon \text { or } \mathcal{B}\left(x_{k}-x_{n}, \beta\right) \geqslant \epsilon, y\left(x_{k}-x_{n}, \beta\right) \geqslant \epsilon\right\}
$$

has $\lambda$-density zero. That is $\delta_{\lambda}\left({ }_{\lambda} K C_{\epsilon}\right)=0$.

Theorem 4.2. If a sequence $x_{k}$ is $\lambda$-statistical convergent in NNS V, then it is $\lambda$-statistical Cauchy.

Proof. Let a sequence $x_{k}$ is $\lambda$-statistical convergent in NNS V. We get $(1-\epsilon) *(1-\epsilon)>1-\mu$ and $\epsilon \diamond \epsilon<\mu$ for a given $\epsilon>0$ and choose $\mu>0$. Then, we have

$$
\delta_{\lambda}(A(\epsilon, \beta))=\delta_{\lambda}\left(k \in I_{n}: \mathcal{G}\left(x_{k}-l, \frac{\beta}{2}\right) \leqslant 1-\epsilon \text { or } \mathcal{B}\left(x_{k}-l, \frac{\beta}{2}\right) \geqslant \epsilon, y\left(x_{k}-l, \frac{\beta}{2}\right) \geqslant \epsilon\right)=0
$$

and so

$$
\left.\delta_{\lambda}\left(A^{c}(\epsilon, \beta)\right)=\delta_{\lambda}\left(k \in I_{n}: \mathcal{G}\left(x_{k}-l, \frac{\beta}{2}\right)>1-\epsilon \text { or } \mathcal{B}\left(x_{k}-l, \frac{\beta}{2}\right)<\epsilon, y\left(x_{k}-l, \frac{\beta}{2}\right)<\epsilon\right)\right)=1
$$

for $\beta>0$. Let $p \in A^{c}(\epsilon, \beta)$, then

$$
\mathcal{G}\left(x_{k}-l, \frac{\beta}{2}\right)>1-\epsilon \text { and } \mathcal{B}\left(x_{k}-l, \frac{\beta}{2}\right)<\epsilon, y\left(x_{k}-l, \frac{\beta}{2}\right)<\epsilon .
$$

Let

$$
B(\epsilon, \beta)=\left\{k \in I_{n}: \mathcal{G}\left(x_{k}-x_{n}, \beta\right) \leqslant 1-\mu \text { or } \mathcal{B}\left(x_{k}-x_{n}, \beta\right) \geqslant \mu, y\left(x_{k}-x_{n}, \beta\right) \geqslant \mu\right\} .
$$


We claim that $B(\epsilon, \beta) \subset A(\epsilon, \beta)$. Let $q \in B(\epsilon, \beta) / A(\epsilon, \beta)$, then

$$
\mathcal{G}\left(x_{q}-x_{n}, \beta\right) \leqslant 1-\mu \text { and } \mathcal{G}\left(x_{q}-l, \frac{\beta}{2}\right)>1-\mu,
$$

in particular $\mathcal{G}\left(x_{n}-l, \frac{\beta}{2}\right)>1-\epsilon$. Then

$$
1-\mu \geqslant \mathcal{G}\left(x_{q}-x_{n}, \beta\right) \geqslant \mathcal{G}\left(x_{q}-l, \frac{\beta}{2}\right) * \mathcal{G}\left(x_{n}-l, \frac{\beta}{2}\right)>(1-\epsilon) *(1-\epsilon)>1-\mu,
$$

which is not possible. Moreover,

$$
\mathcal{B}\left(x_{\mathrm{q}}-x_{n}, \beta\right) \geqslant \mu \text { and } \mathcal{B}\left(x_{\mathrm{q}}-l, \frac{\beta}{2}\right)<\mu,
$$

in particular $\mathcal{B}\left(x_{n}-l, \frac{\beta}{2}\right)<\epsilon$. Then,

$$
\mu \leqslant \mathcal{B}\left(x_{q}-x_{n}, \beta\right) \leqslant \mathcal{B}\left(x_{q}-l, \frac{\beta}{2}\right) \diamond \mathcal{B}\left(x_{n}-l, \frac{\beta}{2}\right)<\epsilon \diamond \epsilon<\mu,
$$

which is not possible. Similarly,

$$
y\left(x_{q}-x_{n}, \beta\right) \geqslant \mu \text { and } y\left(x_{q}-l, \frac{\beta}{2}\right)<\mu,
$$

in particular $y\left(x_{n}-l, \frac{\beta}{2}\right)<\epsilon$. Then,

$$
\mu \leqslant y\left(x_{q}-x_{n}, \beta\right) \leqslant y\left(x_{q}-l, \frac{\beta}{2}\right) \diamond y\left(x_{n}-l, \frac{\beta}{2}\right)<\epsilon \diamond \epsilon<\mu,
$$

which is not possible. In that case $B(\epsilon, \beta) \subset A(\epsilon, \beta)$. Then, by $(4.1), \delta_{\lambda}(A(\epsilon, \beta))=0$, and $\left(x_{k}\right)$ is $\lambda$-statistical Cauchy in NNS.

Definition 4.3. The NNS $V$ is called $\lambda$-statistically complete, if every $\lambda$-statistical Cauchy is $\lambda$-statistical convergent in NNS.

Theorem 4.4. Every neutrosophic normed space $\mathrm{V}$ is $\lambda$-statistically complete.

Proof. Let $x_{k}$ be $\lambda$-statistical Cauchy but not $\lambda$-statistical convergent in NNS. Choose $\mu>0$. We get $(1-\epsilon) *(1-\epsilon)>1-\mu$ and $\epsilon \diamond \epsilon<\mu$, for a given $\epsilon>0$ and $\beta>0$. Since $x_{k}$ is not $\lambda$-statistical convergent in NNS,

$$
\begin{aligned}
& \mathcal{G}\left(x_{k}-x_{n}, \beta\right) \geqslant \mathcal{G}\left(x_{k}-l, \frac{\beta}{2}\right) * \mathcal{G}\left(x_{n}-l, \frac{\beta}{2}\right)>(1-\epsilon) *(1-\epsilon)>1-\mu, \\
& \mathcal{B}\left(x_{k}-x_{n}, \beta\right) \leqslant \mathcal{B}\left(x_{k}-l, \frac{\beta}{2}\right) \diamond \mathcal{B}\left(x_{n}-l, \frac{\beta}{2}\right)<\epsilon \diamond \epsilon<\mu, \\
& y\left(x_{k}-x_{n}, \beta\right) \leqslant y\left(x_{k}-l, \frac{\beta}{2}\right) \diamond y\left(x_{n}-l, \frac{\beta}{2}\right)<\epsilon \diamond \epsilon<\mu,
\end{aligned}
$$

for

$$
P(\epsilon, \mu)=\left\{k \in I_{n}, \mathcal{B}_{x_{k}-x_{n}}(\epsilon) \leqslant 1-\mu\right\} .
$$

So, $\delta_{\lambda}(P(\epsilon, \mu))=1$ which is a contradiction, since $\left(x_{k}\right)$ was $\lambda$-statistical Cauchy in NNS. So that $\left(x_{k}\right)$ must be $\lambda$-statistical convergent in NNS. Hence every NNS is $\lambda$-statistically complete. 


\section{Conclusion}

In this paper, we have defined to neutrosophic normed space and $\lambda$-statistical convergence in neutrosophic normed space. The topological properties of NNSs have been established and examples are given. Further, $\lambda$-statistical convergence with respect to neutrosophic norm is introduced and some fundamental properties are examined. $\lambda$-Statistical Cauchy sequence and $\lambda$-statistically completeness for neutrosophic norm are defined.

\section{Author details}

Vakeel A. Khan received the M.Phil. and Ph.D. degrees in Mathematics from Aligarh Muslim University, Aligarh, India. Currently he is a Associate Professor at Aligarh Muslim University, Aligarh, India. A vigorous researcher in the area of Sequence Spaces, he has published a number of research papers in reputed national and international journals, including Numerical Functional Analysis and Optimization (Taylors and Francis), Information Sciences (Elsevier), Applied Mathematics Letters (Elsevier), A Journal of Chinese Universities (Springer-Verlag, China).

Hira Fatima received B.Sc, M.Sc. and Ph.d. degrees from Aligarh Muslim University, and is currently an Assistant Professor at Mangalayatan University. A vigorous researcher in the area of double sequence spaces, she has published a number of research papers in reputed national and international journals, including Journal of Intelligent and Fuzzy Systems, Filomat, International Mathematical Journal of Analysis and its Applications, etc.

Mohammad Daud Khan received B.Sc and M.Sc degrees from Aligarh Muslim University, and currently he is research Scholar of Dr. Vakeel A. Khan in the department of mathematics, Aligarh Muslim University, Aligarh.

\section{Acknowledgment}

The authors would like to thank the referees and the editor for their careful reading and their valuable comments.

\section{References}

[1] K. T. Atanassov, Intuitionistic fuzzy sets, Physica-Verlag, Heidelberg, (1999). 1

[2] T. Bera, N. K. Mahapatra, On neutrosophic soft linear spaces, Fuzzy Infor. Eng., 9 (2017), 299-324. 1, 2.10

[3] J. Connor, On strong matrix summability with respect to a modulus and statistical convergence, Canad. Math. Bull., 32 (1989), 194-198. 2.7

[4] A. Esi, Asymptotically $\lambda$-invariant statistical equivalent sequences of fuzzy numbers, Math. Sci. (Springer), 6 (2012), 5 pages. 1,2

[5] A. Esi, N. Braha On asymptotically $\lambda$-statistical equivalent sequences of interval numbers, Acta Sci. Tech., 35 (2013), 515-520. 1

[6] A. Esi, B. Hazarika, ג-ideal convergence in intuitionistic fuzzy 2-normed linear space, J. Intell. Fuzzy Systems, 24 (2013), 725-732. 1

[7] M. Et, M. Çınar, M. Karakaş, On $\lambda$-statistical convergence of order $\alpha$ of sequences of function, J. Inequal. Appl., 2013 (2013), 8 pages. 2.4

[8] H. Fast, Sur la convergence statistique, Colloq. Math., 2 (1951), 241-244. 1

[9] J. A. Fridy, On statistical convergence, Analysis, 5 (1985), 301-314. 1

[10] A. George, P. Veeramani, On some results in fuzzy metric spaces, Fuzzy Sets and Systems, 64 (1994), 395-399. 1

[11] E. Kolk, On strong boundedness and summability with respect to a sequence of modulii, Acta Comment. Univ. Tartu, 960 (1993), 41-50. 2.7

[12] K. Menger, Statistical metrics, Proc. Nat. Acad. Sci. U.S.A., 18 (1942), 535-537. 2

[13] J. H. Park, Intuitionistic fuzzy metric spaces, Chaos Solitons Fractals, 22 (2004), 1039-1046. 1

[14] R. Saadati, J. H. Park, On the intuitionistic fuzzy topological spaces, Chaos Solitons Fractals, 27 (2006), 331-344. 2.8, 2.9

[15] F. Smarandache, Neutrosophic set, a generalization of intuitionistic fuzzy sets, Int. J. Pure Appl. Math., 24 (2005), 287-297. 1

[16] L. A. Zadeh, Fuzzy sets, Inf. Control, 8 (1965), 338-353. 1 